\title{
Group-Level Events are Catalysts in the Evolution of Cooperation
}

\author{
Burton Simon ${ }^{1}$ and Michael Pilosov ${ }^{2}$
}

\begin{abstract}
Group-level events, like fission and extinction, catalyze the evolution of cooperation in group-structured populations by creating new paths from uncooperative population states to more cooperative states. Group-level events allow cooperation to thrive under unfavorable conditions such as low intra-group assortment and moderate rates of migration, and can greatly speed up the evolution of cooperation when conditions are more favorable. The timedependent effects of fission and extinction events are studied and illustrated here using a PDE model of a group-structured population based loosely on populations of hunter-gatherer tribes. By solving the PDE numerically we can compare models with and without group-level events, and explicitly calculate quantities associated with dynamics, like how long it takes a small population of cooperators to become a majority, as well as equilibrium population densities.
\end{abstract}

\section{Introduction}

In mathematical models of evolutionary processes, cooperation is characterized by behaviors that are detrimental to the individual, but advantageous to the individual's social partners, e.g., Nowak, 2006. Here, we will call individuals that exhibit cooperative behaviors cooperators, and those that do not will be called defectors. Cooperative behaviors are very common in the natural world, but in general their evolution is more difficult to explain than the evolution of traits or behaviors that more directly benefit the individual, Darwin, 1859, 1871; Hamilton, 1964; Dawkins, 1976; Maynard Smith and Szathmary, 1997; Sober and Wilson, 1998; Okasha, 2006; Nowak, 2006; Fletcher and Doebeli, 2009; Tarnita, et.al., 2013. Mathematical explanations of the evolution of cooperation based on kin selection theory date back to Fisher, 1930; Haldane, 1932; Hamilton, 1964; and Price, 1972, but the basic ideas were anticipated much earlier, e.g. Darwin, 1859, who sought to explain the existence of sterile castes of social insect. Mathematical explanations

\footnotetext{
${ }^{1}$ Department of Mathematical and Statistical Sciences, University of Colorado Denver, burt. simon@ucdenver.edu

${ }^{2}$ Department of Mathematical and Statistical Sciences, University of Colorado Denver, michael.pilosoveucdenver.edu
} 
of the evolution of cooperation based on group selection theory date back to Maynard Smith, 1964, 1976; Gilpin, 1975; and Wilson, 1975, but the basic ideas here also date back to Darwin, 1871, who wondered if competition between tribes of humans in the past may explain modern human cooperation. Since the 1960s, both kin selection theory and group selection theory have gone through a number of changes and generalizations, Wynne-Edwards, 1962; Williams, 1966; Maynard Smith, 1964, 1976; Price, 1972; Wilson, D.S., 1975; Wilson, E.O., 1975; Dawkins, 1976; Damuth and Heisler, 1988; Goodnight, Schwartz and Stevens, 1992; Sober and Wilson, 1998; Wilson and Wilson, 2007; Gardner, West and Wild, 2011; Frank, 2012, 2013; Simon, Fletcher and Doebeli, 2013. Here we take a group selection approach to the evolution of cooperation, and in particular, we use the definitions of group selection and related concepts proposed and defended in Simon, Fletcher and Doebeli, 2013, which can be summarized for our current purposes as follows:

Definition: Group-level events include all biological events that change the number of groups in a population. ${ }^{3}$ A trait evolves by group selection in a model of a group-stuctured population if it establishes itself when group-level events are present in the model, and does not establish itself when they are absent. In other words, an individual-level trait (like cooperation) evolves by group selection in a model if the group-level events are decisive, in the sense that when the other model parameters are held fixed, the trait evolves if and only if the group-level events occur frequently enough.

A trait that does not (strictly) evolve by group selection may still be assisted by group-level events, for example if it establishes itself more quickly and/or more completely when group-level events are present in the model than when they are absent.

The primary reason biologists study group selection is the theoretical possibility that a trait that is disadvantageous for individuals within their groups, can nevertheless thrive in the population

\footnotetext{
${ }^{3}$ Some events that cause abrupt changes in the state of the population are also group-level events, even if the number of groups does not change. For example, an event where a group dies and another is born, simultaneously, is a group-level event. See Simon, Fletcher and Doebeli, 2013 for formal definitions of group-level and individual-level events.
} 
because groups containing more individuals with the trait (or a larger fraction with the trait) are endowed with some kind of advantage over other groups. The nature and strength of this grouplevel advantage, and its cause, is not the same in every model. For example, in many models, abundance of the trait in a group is correlated with a faster group growth rate (i.e., higher MLS1 fitness, Okasha, 2006), which can lead to an increase of the trait in the population. This is the kind of group selection that is often said to be mathematically equivalent to kin selection, e.g., Gardner, et.al., 2011. However, this is not the kind of group selection we are talking about here, since group-level events in those models may not be decisive, or may be absent altogether. A faster group growth rate from an abundance of a trait is neither necessary nor sufficient for group selection to occur. (See Simon, Fletcher and Doebeli, 2013 for an example of group selection where groups with more of a cooperative trait actually grow slower.) The kind of group selection we are talking about here occurs when groups gain their advantage directly or indirectly from the effects of group-level events, e.g., a lower extinction rate for groups with an aboundance of the trait. There is no ambiguity in this definition because the advantage can be measured by solving the model with and without the group-level events. Maynard Smith, 1976, described a similar kind of group selection in words, but his models did not include realistic group-birth events, like fission. Since fission events create much of the group-level diversity that the group extinction events select on, his models underestimated the strength of group selection.

The analysis here is not the first in the literature to study the effects of fission and extinction on group-structured populations mathematically. Previous work using continuous-time Markov chains and/or PDE models includes Simon, 2010, 2014; Simon and Nielsen, 2012; Simon, Fletcher and Doebeli, 2012, 2013; Luo, 2013; van Veelen, Luo and Simon, 2014. Previous to that body of work, Traulsen and Nowak, 2006, and Lehmann, et.al., 2007, studied a group-structured population of cooperators and defectors with the same biological events considered here (births, deaths, migration, fission, extinction), and found conditions for cooperators to be more likely to fixate than defectors, starting with a single mutant. Traulsen and Nowak used a multi-level selection approach to the problem (nested Moran processes). Lehmann, et.al. used a kin selection approach to the 
same basic model, showing that at least some aspects of the model could be studied either way. Both papers found that group-level events promote cooperation, in the sense that without them, defectors are always more likely to fixate. The analytical results in both papers are restricted to limiting versions of the basic model, where the fission, extinction, and migration rates converge to zero, and where there is weak selection between cooperators and defectors within the groups. (Traulsen and Nowak obtain results when fission, extinction, and migration rates are nonzero by simulation, but they assume weak selection throughout.) In this paper, and with PDE models in general, there are no restrictions on any of the model parameters, as long as the resulting rate functions are nonnegative and differentiable in the population variables. In particular, there is no need to assume constant populations. Thus, the scope of the PDE model is much larger. However, the most significant differences between the analyses in Traulsen and Nowak, and Lehmann, et.al., and the present work have to do with dynamics. Those analyses are insensitive to the time scales and trajectories of the evolutionary processes, and therefore cannot address the main questions posed here, which involve rates of evolutionary change. Furthermore, fixation is not the criteria for evolutionary success here. In fact, fixation does not occur in general in PDE models of group-structured populations, Simon, 2010; Simon, Fletcher and Doebeli, 2013; Luo, 2013. When the rates of group-level events are positive, the equilibrium typically is a smooth density function corresponding to a diverse population of groups of varying sizes and compositions. Diversity in equilibrium has been observed before in models of cooperators and defectors, e.g. Doebeli, Hauert and Killingback, 2004; Archetti and Scheuring, 2010. Of course, fixation theoretically occurs in finite time in any finite population, since the population state space is finite and fixations are the only absorbing states. But the PDE models suggest that the time to fixation may be very large in group-structured populations with group-level events, especially when populations (of groups and individuals) are large, so fixation probabilities may not always be the appropriate way to characterize the evolution of cooperation in group-structured populations.

Likewise, the static analysis of a group-structured model with fission events (referred to as budding) in Gardner and West, 2006 is not sensitive to the kinds of dynamical effects and equilib- 
rium outcomes found here, although it does find that budding benefits cooperation. Theoretically, it might be possible to obtain equilibrium population densities numerically for our PDE models by a static analysis (by setting the time derivative to zero, and solving the resulting equation), but this has not yet been demonstrated, and even if equilibrium densities can be found this way, important questions concerning evolutionary rates and trajectories would remain unanswered.

Of course, group selection is not the whole story in the evolution of cooperation. The definition of group selection we use here does not preclude the possibility that other factors are decisive (or assist) in the evolution as well. The evolution of cooperation can be the net result of multiple factors. In particular, it is well known that if cooperators (defectors) are more likely to interact socially with other cooperators (defectors) than pure chance would allow, then cooperation typically evolves more easily in the model. This is one of the basic insights of kin selection theory, Hamilton, 1964. Thus, the level of assortment within the groups (which quantifies the social interaction asymmetry in our model) may also be decisive. Comparisons between group selection and kin selection, as explanations of the evolution of cooperation in a given model, are (or should be) about the relative efficacy of model variables like the level of assortment within the groups and the rates that group-level events occur. In fact, it is apparent from the analysis here that group-level events and intra-group assortment work together synergistically to enable the evolution of cooperation.

There are quite a few parameters that can be varied in our hunter-gatherer model (Table 1), however we will focus primarily on three model parameters in our analysis.

$\rho:$ a linear scaling parameter that controls the rates that fission and extinction events occur.

$r$ : a measure of assortment within the groups.

$\mu$ : the per-capita migration rate.

Thus, the effects of group-level events can be studied by varying $\rho$. (When $\rho=0$ there are no group-level events.) By varying $r$ we can study the effects of assortment, and by varying $\mu$ we can study the effect of migration. We also study the effects of selective extinction (when morecooperative groups have lower extinction rates), Haldane, 1932; Aoki, 1982; Bowles, 2006, and 
associative splitting (when fission events segregate cooperators and defectors to some degree), Haldane, 1932; Wright, 1943; Hamilton, 1975; Eshel and Cavalli-Sforza, 1982; Bowles, 2006. We find that while selective extinction and associative splitting can be beneficial to the evolution of cooperation, neither is decisive in general. A thorough analysis of the evolution of cooperation over the whole set of feasible parameter values would be impractical, and is not the object of this paper.

The range of parameter values studied here are intended to resemble those of ancient huntergathers qualitatively, if not quantitatively. Estimates of the parameters we would need to specify a quantitatively accurate model of hunter-gatherers (ancient or more modern), especially those parameters pertaining to group-level event rates, are not available in the anthropological literature, Yellen and Harpending, 1972; Birdsell, 1953; Bowles, 2006; R. Bettinger and P. Richerson, private communication. Therefore our numerical results are only suggestive. However, our results show in great detail the kinds of the things that can be learned from PDE models of group-structured populations. For example, in our baseline model of hunter-gatherers (Table 1), our calculations show that a small population of cooperators (about $5 \%$ of the population) grows to $50 \%$ of the population in about 5000 years (Figure 3a, $r=.15, \rho=1$ ). Without group-level events, the same small population of cooperators goes extinct. If the within-group assortment is increased in the baseline model to $r=.30$ then cooperation increases to $50 \%$ of the population even in the absense of group-level events (Figure 3a, $r=.30, \rho=0$ ). However with the baseline rate of group-level events $(\rho=1)$, the time it takes to reach $50 \%$ decreases by a factor of three (from about 6000 years to about 2000 years).

The present modeling framework can address the following kinds of questions in the context of a model of hunter-gatherers.

- For which model parameter settings does cooperation thrive in equilibrium?

- How long does it take for cooperation to establish itself in the population (e.g., to reach $50 \%$ of the population), when it does thrive? 
- How much faster is the evolution of cooperation when group-level events occur, in cases where cooperation would evolve even without them?

- How often do group-level events need to occur for cooperation to thrive, in cases where the intra-group assortment is insufficient (on its own) to support cooperation?

- How much intra-group assortment is needed for cooperation to thrive, in cases where the group-level event rates are insufficient (on their own) to support cooperation?

- How much does migration slow down the evolution of cooperation, and how much migration can occur before cooperation can no longer thrive?

- Which rates of group-level events lead to the largest equilibrium populations of individuals and groups?

- How do the evolutionary trajectories and equilibrium densities differ with different rates of group-level events, different levels of assortment, etc.?

In the next section we describe a generic PDE model, and then assign values (or ranges) to the parameters corresponding to our baseline hunter-gatherer model. Section 3 lists five general observations about the hunter-gatherer model that follow from our numerical experiments. In section 4 we conjecture that the cooperator-friendly effects of group-level events in our huntergatherer model occur much more generally, and briefly discuss applications of the techniques used here to other group-structured populations. There are links to a Matlab program in the Methods section that can solve our baseline model, and in fact any instance of our PDE model. There are also links to full numerical animations of solutions of our PDE model, which are more informative than the still figures in the main text. 


\section{The PDE Model of Hunter-Gatherer Tribes}

We begin by describing a generic PDE model of group-structured populations of cooperators and defectors, and then choose parameter values in ranges suggested by populations of hunter-gatherer tribes in order to study that region of the parameter space in detail.

\subsection{A Generic PDE Model of Group-Structured Populations}

The PDE model we use here to study group-structured populations is a continuous-state approximation of a certain discrete-state Markovian model of group-structured populations, Simon, 2010. The population in the discrete model consists of groups comprised of cooperators and defectors. There are births and deaths of individuals within the groups, and there are births and deaths of groups via fission and extinction events. There are also migrations of individuals between groups. The states of the discrete Markovian models specify the number of $(x, y)$-groups ${ }^{4}$ in the population, where $x$ and $y$ range over the nonnegative integer lattice. The evolutionary trajectories of a properly constructed sequence of scaled ${ }^{5}$ Markovian models, with increasing populations, will converge to the solution of the PDE. The mathematics that precisely describes and justifies the convergence is beyond the scope of this paper. Proofs of large-population limit theorems for models similar to ours here can be found in Champagnot, et.al, 2006; Puhalskii and Simon, 2012; Luo, 2013; Luo and Mattingly, 2015.

The PDE that arises in the limit has the form

$$
\frac{\partial \theta_{t}}{\partial t}+\frac{\partial\left(\theta_{t} \alpha_{t}^{c}\right)}{\partial x}+\frac{\partial\left(\theta_{t} \alpha_{t}^{d}\right)}{\partial y}=g_{t}
$$

where $\theta_{t}(x, y)$ is the population state variable, interpreted as the number of $(x, y)$-groups in the population at time $t$. In the PDE model, $x$ and $y$ are real-valued, and not integer-valued. Intuitively,

\footnotetext{
${ }^{4} \mathrm{An}(x, y)$-group is a group with $x$ cooperators and $y$ defectors. Markovian processes like these are often referred to as measure-valued Markov processes, Luo and Mattingly, 2015, since the most natural way to represent the state is as an integer-valued measure on the nonnegative quadrant.

${ }^{5}$ By a scaling we mean that the parameters specifying the $n$th Markov process in the sequence are specially chosen functions of $n$, and that the population variables are also scaled in such a way that they converge to finite limits.
} 
an $(x, y)$-group can simply be thought of as a group with an integer number of cooperators and defectors with values close to $(x, y){ }^{6}$

Under the scaling that leads to the PDE limit, there is usually either zero or one $(x, y)$-group in the population. One can therefore visualize a state of the Markovian process as a collection of dots on the nonnegative quadrant of the $(x, y)$ plane, where each dot corresponds to a group. The position of the group reveals its state, i.e., a dot at $(x, y)$ corresponds to an $(x, y)$-group. When the state of a group changes by one unit (due to a birth, death, or migration), the corresponding dot moves. When a group dies of extinction, the dot disappears. When a group fissions, the dot disappears, and new dots (closer to the origin) appear, corresponding to the fissioned pieces. Simon and Nielsen, 2012 have animations of simulations of this process which are very useful for understanding the PDE model.

In (1), the functions $\alpha_{t}^{c}(x, y)$ and $\alpha_{t}^{d}(x, y)$ characterize the cooperator/defector population dynamics within the groups, including the effects of migration. The right side term, $g_{t}(x, y)$, characterizes the effects of all the group-level events, which involve more abrupt changes to the state of the population, i.e., births and deaths of groups. The functions $\alpha_{t}^{c}(x, y), \alpha_{t}^{d}(x, y)$, and $g_{t}(x, y)$ are constructed from the rate functions for the five biological events featured in the Markovian model. Thus, one can expect that the PDE model will behave analogously to the Markovian model it is designed to approximate. Animations of solutions of the PDE (see Methods section) look very much like the animations of the Markovian trajectories, ${ }^{7}$ as one would expect. The advantage of studying the PDE model, and not the underlying Markovian model, is that the PDE is a deterministic equation which can be solved numerically. (See Methods section.) The only way to analyze the underlying Markovian model is by (Monte Carlo) simulation since it has such a complicated structure. The functions $\alpha_{t}^{c}(x, y), \alpha_{t}^{d}(x, y)$, and $g_{t}(x, y)$ will be explicitly constructed for the hunter-gatherer example next.

\footnotetext{
${ }^{6}$ Continuous-state models like these are often referred to as "infinite population" models. It is much better to think of them as continuous approximations of finite discrete models, justified by large population limits.

${ }^{7}$ Eventually the discrete models get trapped in an absorbing class of states (fixation), so the similarity does not last indefinitely.
} 


\subsection{The Hunter-Gatherer Model}

By the time hunter-gatherer societies were being studied scientifically by anthropologists, they had already changed profoundly from their original states due to disease and economic partnerships resulting from contact with modern societies. However, there are studies that shed light on how a mathematical model of hunter-gatherer populations might be structured, e.g., Birdsell, 1953; Yellen and Harpending, 1972; Bettinger, 1991; Bowles, 2006. Of most relevance here is the heirarchical structure that many of these populations share. The individuals, or course, are the human hunter-gatherers. Individuals live in families that may forage together, apart from other families, but a collection of family groups, numbering perhaps 50 individuals, form "bands" that constitute the primary social groups. A number of bands, often speaking similar dialects, come together occasionally (e.g. anually) for celebrations that may involve finding mates from other bands. These "tribes" may number 500 individuals or more, and of course, there may be a number of tribes in the larger population. At the band and tribal level, fission occurs when the size of the unit increases much beyond its preferred state, and of course extinction of group-level units due to disease or battles occurs sometimes as well. (R. Bettinger and P. Richerson, private communication.) For our purposes here, we will consider the bands to be the the groups in the model, and a single tribe to be the whole population. Thus, the exchanging of mates between bands during celebrations is migration in the model, and is not negligable. See Appendix A2 for a discussion of how the model parameters were set. Note that an alternate modeling approach would be to consider the tribes to be the groups, or even to consider a three-level process with individuals, bands, and tribes; but these ideas will not be pursued here.

Our hunter-gatherer model is a special case of the generic model just described. From equation (1) we see that the dynamics are governed by the functions $\alpha_{t}^{c}(x, y), \alpha_{t}^{d}(x, y)$ and $g_{t}(x, y)$, which

can be derived from the rate functions for the five events in the model. We therefore begin by specifying the rate functions for our hunter-gatherer model.

The cooperator/defector population dynamics within the groups includes a rule that determines the level of assortment within the group. The rule is that when cooperators (defectors) choose 
a partner to interact with, it is a cooperator (defector) with probability $r$, and someone chosen randomly from the group with probability $1-r$. The resulting evolutionary process is similar to ones described in Eshel and Cavalli-Sforza, 1982; Grafen, 1985; Fletcher and Doebeli, 2009, van Veelen, et.al., 2012; Allen, et.al., 2013; Allen and Nowak, 2015. A simple calculation shows that for every group, regardless of its state, the probability a cooperator is paired with a cooperator minus the probability a defector is paired with a cooperator is always $r$. (See equation (3) below.) Our level of assortment is therefore equivalent to the usual definition of relatedness used in the quantitative genetics literature, applied to one of our groups in isolation, van Veelen, et. al., 2014. However, the variable $r$ is not the same as the level of assortment (or coefficient of relatedness) for the whole population, $R_{t}$, which is a time-varying, emergent property of the two-level population process that can only be determined by solving the model. (See Simon, Fletcher and Doebeli, 2012, or equation (15) in the Appendix.)

Birth rates at time $t$ for the cooperators and defectors in a given group are proportional to their expected payoffs from a prisoner's dilemma game with payoff matrix

$$
\left[\begin{array}{ll}
R & S \\
T & P
\end{array}\right]=\left[\begin{array}{cc}
1+b-c & 1-c \\
1+b & 1
\end{array}\right]
$$

Parameters $b$ and $c$ in the payoff matrix can be interpreted as the benefit and cost associated with cooperation.

Individuals play Prisoner's Dilemma with their group-mates, but the pairings are not completely random. A parameter $r \in[0,1]$ controls the assortment. In particular, an individual chooses a partner of the same type (cooperator or defector) with probability $r$, and chooses a partner randomly from the whole group with probability $1-r$. In an $(x, y)$-group (a group with $x$ cooperators and $y$ defectors) it follows that the probability a cooperator is paired with a cooperator and a defector is paired with a cooperator are (respectively)

$$
P(C \mid C)=r+(1-r) \frac{x}{x+y}, \quad \text { and } \quad P(C \mid D)=(1-r) \frac{x}{x+y}
$$


The expected payoffs for cooperators and defectors in an $(x, y)$-group are therefore

$$
\beta_{c}(x, y)=r(1+b-c)+(1-r)\left(\frac{x}{x+y}(1+b-c)+\frac{y}{x+y}(1-c)\right)
$$

and

$$
\beta_{d}(x, y)=r+(1-r)\left(\frac{x}{x+y}(1+b)+\frac{y}{x+y}\right) .
$$

The per capita birth rates of cooperators and defectors in an $(x, y)$-group are set to $s \beta_{c}(x, y)$ and $s \beta_{d}(x, y)$, where $s>0$ is a scaling parameter. The per capita death rates for cooperators and defectors in an $(x, y)$-group are equal and proportional to the size of the group, i.e.

$$
\delta_{c}(x, y)=\delta_{d}(x, y)=d(x+y),
$$

where $d>0$ is the death rate parameter. Note that the per capita birth rate functions are bounded by $s(1+b)$, but the per capita death rates are unbounded in the size of the group. It is reasonable to have death rates grow without bound since there are limited resources the individuals must share, however, the simple linear form of the death rate function was chosen primarily for convenience. We show in the next subsection how the birth and death rate parameters determine the average lifespans of the individuals, and the equilibrium group sizes.

There is also migration in the model. Each individual migrates from its group at rate $\mu$ and joins another group at random. Let $C_{t}$ and $D_{t}$ denote the total number of cooperators and defectors in the population at time $t$ (summed over all the groups), and let $G_{t}$ be the number of groups. These values can be calculated from the state of the population, $\theta_{t}(x, y)$,

$$
G_{t}=\iint \theta_{t}(x, y) d y d x, \quad C_{t}=\iint x \theta_{t}(x, y) d y d x, \quad D_{t}=\iint y \theta_{t}(x, y) d y d x
$$

It follows that the total rate that cooperators migrate away from an $(x, y)$-group is $\mu x$, and the rate that cooperators migrate to a given group is $\mu \frac{C_{t}}{G_{t}}$. (Note: $\frac{C_{t}}{G_{t}}$ is the average number of cooperators per group.) Likewise defectors migrate away from an $(x, y)$-group at rate $\mu y$ and migrate into it 
at rate $\mu \frac{D_{t}}{G_{t}}$. The within-group population dynamics in our model are therefore governed by the differential equations

$$
\left[\begin{array}{l}
x^{\prime} \\
y^{\prime}
\end{array}\right]=\left[\begin{array}{c}
\left(s \beta_{c}(x, y)-\delta_{c}(x, y)\right) x+\mu\left(\frac{C_{t}}{G_{t}}-x\right) \\
\left(s \beta_{d}(x, y)-\delta_{d}(x, y)\right) y+\mu\left(\frac{D_{t}}{G_{t}}-y\right)
\end{array}\right] \equiv\left[\begin{array}{c}
\alpha_{t}^{c}(x, y) \\
\alpha_{t}^{d}(x, y)
\end{array}\right] .
$$

The ODEs that govern the within-group population dynamics are exactly the functions that show up in the partial derivative terms of (1) (explained in Simon, 2010). In Appendix A1 we derive Hamilton's rule for an isolated group when the population dynamics is governed by (8) with $\mu=0$ (no migration).

At the group level, the population is governed by a fission-growth-extinction process (Figure 1). The growth aspect of the group-level population process is the result of births, deaths, and migrations. Groups grow (or shrink) and change in composition (plus or minus one individual at a time) over time due to individual-level events. (In the PDE limit, these changes are continuous.) Likewise, individuals are affected by group-level events. When a fission event occurs, the affected individuals suddenly find themselves in a group that may be very different in size and composition from the one they were in before, and of course individuals die in group extinction events. The time-varying rates that individual-level and group-level events occur are therefore highly interdependent.

Fission rates in our model are chosen so that larger groups are more likely to fission than smaller ones. The class of fissioning densities (described below) used in the model allows cooperators and defectors to "segregate" to some extent during a fission event. This is sometimes referred to as “selective assortment" or "associative (tribe) splitting”, Haldane, 1932; Wright, 1943; Aoki, 1982; Bowles, 2006.

In our model, an $(x, y)$-group fissions at rate

$$
f(x, y)=\lambda(x+y)
$$


where $\lambda \geq 0$ is the fission rate parameter. When a group fissions the outcome is specified (statistically) by a fissioning density, Simon, 2010. A fissioning density is a function $h((x, y),(u, v))$ where $h((x, y),(u, v)) d y d x$ is interpreted as the expected number of $(x, y)$-groups resulting from the fissioning of a $(u, v)$-group. For $(u, v)$ fixed, $h((x, y),(u, v))$ is a function with support on $[0, u] \times[0, v]$, whose integral over that region is equal to 2 (since a fission event results in two pieces), and whose mean is not greater than $(x / 2, y / 2)$. (If the mean is less than $(x / 2, y / 2)$, then some individuals die in the fission event. Here we assume the mean equals $(x / 2, y / 2)$.) The fissioning density used in our hunter-gatherer model is a weighted average of a uniform density and a discrete density, yielding a simple way to include associative splitting. The uniform part of the density sets all possible outcomes of the fissioning of an $(x, y)$-group to be equally likely, i.e. $h_{1}((x, y),(u, v))=\frac{2}{u v}$. The discrete part of the density is $h_{2}((x, y),(u, v))=\delta_{((x, y)=(0, v / 2))}+\delta_{((x, y)=(u, v / 2))}$, where $\delta_{(\cdot)}$ is the delta function. In other words, all the cooperators are in one piece, while the defectors split evenly between the two pieces. The fissioning density is

$$
h((x, y),(u, v))=(1-\psi) h_{1}((x, y),(u, v))+\psi h_{2}((x, y),(u, v)),
$$

where $\psi \in[0,1]$ is the associative splitting parameter. When $\psi=0$ there is no associative splitting, so the outcomes of fission events are random (uniform). When $\psi=1$, the fission density is $h_{2}$. Note that an even more extreme version of associative splitting would have all the cooperators end up in one piece and all the defectors end up in the other, i.e. $h((x, y),(u, v))=\boldsymbol{\delta}_{((x, y)=(u, 0))}+\boldsymbol{\delta}_{((x, y)=(0, v))}$.

The extinction rate function in our model increases with the total number of groups in the population, decreases with the size of the group, and decreases with the prevalence of cooperators in the group, i.e. "selective extinction" (Haldane, 1932; Aoki, 1982; Bowles, 2006). The extinction rate in the model is

$$
e(x, y, G)=\frac{e_{0} G}{(\phi x+y)^{2}}
$$

where $e_{0} \geq 0$ is the extinction rate parameter, and $\phi$ is the selective extinction parameter. Thus, smaller groups are more likely to die, and groups are more likely to die when there are more groups 
to compete with. If $\phi=1$ then the extinction rate depends on the size of the group, but does not distinguish between cooperative and uncooperative groups, i.e. there is no selective extinction. If $\phi>1$ then cooperative groups are favored, if $\phi<1$ then uncooperative groups are favored.

Finally, the initial condition for the population is based on a bivariate Normal density centered at $\left(\bar{C}_{0}, \bar{D}_{0}\right)$,

$$
\theta_{0}(x, y)=\frac{N_{0}}{2 \pi v_{0}} e^{\left(\left(x-\bar{C}_{0}\right)^{2}+\left(y-\bar{D}_{0}\right)^{2}\right) / 2 v_{0}}, \quad(x, y) \geq 0 .
$$

The density is truncated off the positive quadrant, so the initial population $G_{0}=\iint \theta_{0}(x, y) d y d x$, will be less than $N_{0}$, especially if $\left(\bar{C}_{0}, \bar{D}_{0}\right)$ is close to one of the axes. The parameter $v_{0}$ controls the spread of the initial density.

Solving the model means solving equation (1) using $\alpha_{t}^{c}(x, y)$ and $\alpha_{t}^{d}(x, y)$ from (8), and

$$
g_{t}(x, y)=\rho\left(-f(x, y)-e\left(x, y, G_{t}\right)+\int_{x}^{\infty} \int_{y}^{\infty} \theta_{t}(u, v) f(u, v) h((x, y),(u, v)) d v d u\right)
$$

where the first two terms in the parentheses are the fission and extinction rates from (9) and (11), and the third term is the rate that new $(x, y)$-groups are born due to larger groups fissioning. The parameter $\rho$ scales the group-level event rates together. Even in the simplest examples there is no closed-form solution for (1), but the equation can be solved numerically without much difficulty. (See Methods section.) A single run of our numerical solver yields $\theta_{t}, t \in[0, T]$. The trajectories of $\theta_{t}$ converge to an equilibrium, so one chooses a suitably large $T$ and uses $\theta_{T}$ to approximate the equilibrium population density. The Matlab program we used for our numerical experiments is linked to in the Methods section.

Table 1 lists the benchmark (default) values for our hunter-gatherer model. In the figures we allow $r, \rho, \mu, \phi$, and $\psi$ to vary. It turns out that $G_{0}$, the initial number of groups is 384.75 in all our examples and not $N_{0}=471.25$. The reason for this is that since the initial group mean is so close to the $y$ axis, a sizable fraction of the initial density is truncated. The reader may be curious how the benchmark parameter settings were found. We used reasonable estimates of quantities, like average human lifespans, equilibrium group sizes, average times between fission events, etc., 
Table 1: Model Parameters and their Benchmark Values

\begin{tabular}{cll}
\hline \hline Parameter & Description & Benchmark Value \\
\hline$r$ & intra-group relatedness & 0.15 \\
$\rho$ & group-level event rate scaling factor & 1.0 \\
$b$ & benefit associated with cooperation & 0.4 \\
$c$ & cost associated with cooperation & 0.1 \\
$d$ & individual death rate parameter & 0.0005 \\
$s$ & game payoff to birth rate scaling factor & 0.025 \\
$e_{0}$ & extinction rate scaling factor & 0.025 \\
$\phi$ & selective extinction parameter & 1.0 \\
$\lambda$ & fission rate scaling factor & 0.00025 \\
$\psi$ & associative splitting parameter & 0.0 \\
$\mu$ & per individual migration rate & 0.0025 \\
$N_{0}$ & initial number of groups & 471.25 \\
$\left(\bar{C}_{0}, \bar{D}_{0}\right)$ & initial mean group type & $(2,48)$ \\
$v_{0}$ & initial group type variance & 5.0 \\
\hline
\end{tabular}

and found parameter settings that yielded populations consistent with those estimates, using the simple heuristic calculations described in Appendix A2. The heuristics help explain intuitively (if not exactly) how the model variables control the population dynamics.

\section{Results}

The main conclusions from our numerical experiments on the hunter-gatherer model can be summarized as follows:

\section{Group-level events catalyze the evolution of cooperation by creating new paths to the} cooperative states.

When there are no group-level events $(\rho=0)$, cooperation evolves if the level of assortment within the groups is high enough, $r>c / b$, which is Hamilton's rule applied to an isolated group (Figure 2, row 1). Thus, when $r>c / b$, group-level events are not strictly necessary for cooperation to evolve. However, fission events produce a diverse population of group types (Figure 2, row 2) which the extinction process can select against, and cooperation establishes itself in much less time. In this case the catalytic effect speeds up the evolution that would 
have occurred anyway.

When $r<c / b$, cooperation cannot evolve without group-level events, but in our experiments there is a wide range of $\rho$ values where cooperation thrives in equilibrium (Figure 3). In this case the catalytic effect allows cooperation to evolve when it otherwise could not. The catalytic effect of group-level events is strong enough in our model so that cooperation can sometimes evolve even when $r=0$ (Figure 3, blue curves).

\section{Group-level events can be powerful catalysts even without an obvious source of selective extinction and associative splitting.}

Early theorists like Fisher, 1930 and Haldane, 1932 thought that fission and extinction events would enable the evolution of cooperation through selective extinction and associative splitting. The results here show that fission and extinction events can have a profound effect even when their rate functions are symmetric with respect to cooperators and defectors, i.e. with no obvious source of selective extinction or associative splitting, Figure 3a. Adding some selective extinction and associative splitting via the parameters $\phi$ and $\psi$ can have a positive effect on the evolution of cooperation (Figure 3b-d) but the effect is not very large. One explanation for this (perhaps) unexpected result resides in the interplay between (individuallevel) birth rates and (group-level) extinction rates. In the hunter-gatherer model, small cooperative groups grow faster than small uncooperative groups, and are therefore more likely to escape the extinction process, which disproportionately targets small groups (of any composition). Of course, this net result of the interplay between biological events at the individual and group levels amounts to a kind of selective extinction. The nature of the fission and extinction processes also explains the counter-intuitive result that high levels of associative splitting (e.g. using $h((x, y),(u, v))=\delta_{((x, y)=(u, 0))}+\boldsymbol{\delta}_{((x, y)=(0, v))}$ can slow or stop the evolution of cooperation, Figure 4. One reason is that when cooperators are rare, near-perfect segregation leads to cooperators finding themselves in very small groups, which are prone to extinction. Another reason is that when groups are (nearly) monomorphic, a 
high level of associative splitting is not an efficient method of group reproduction. In our hunter-gatherer model, cooperators need the support of defectors to form viable groups, until they establish themselves in the population. In general, it is the group-level diversity caused by (random) fission events that primarilly drives the evolution of cooperation in the hunter-gatherer model.

\section{Group-level events can counter a moderate migration rate.}

Intuitively, the reason why migration undermines the evolution of cooperation in groupstructured populations, like hunter-gatherers, is that the occasional cooperative group that emerges will be "contaminated" by migrating defectors, whose descendants will dominate the group. Mutant defectors born in a cooperative group would have the same effect. However, the nullifying effect of migration (or mutation) has not been studied before in a dynamical model of group-structured populations with significant rates of fission, extinction, and migration. Our results imply that the nullifying effects are sometimes overstated. Figure 5 shows how the migration rate affects the evolution of cooperation for various settings of the level of assortment and group-level event rates. When $r<c / b$ (panels (a) and (b)) there is a critical migration rate (depending on $r$ and $\rho$ ) above which cooperation cannot evolve. If $r \geq c / b$ (panels (c) and (d)) then migration slows down the evolution of cooperation, but cannot stop it. The effects of migration have been studied previously in classical models by considering how it effects relatedness and kin competition, e.g., Lehmann and Rousett, 2010.

\section{Fixation (of cooperators or defectors) does not necessarily occur in group-structured populations.}

In the absence of group-level events, the population equilibrium in our model consists of either all cooperators or all defectors, depending on whether or not Hamilton's rule is satisfied in isolated groups. However, when $\rho>0$, the typical equilibrium is a complicated mixture of groups of various sizes and levels of cooperation, e.g. Figure 2, row 2, or see Simon, 2010, 
or Simon, Fletcher and Doebeli, 2013. Defectors can often persist at small but stable levels even when conditions favor cooperators, e.g., Figure 2, row 2. Likewise, a small population of cooperators can remain in equilibrium even when defectors dominate the population.

\section{Too many group-level events can be harmful.}

The model confirms that if $\rho$ is too big, groups fission into smaller and smaller pieces which are then prone to extinction. The result is a population reduced to small groups (reletive to their equilibrium size), that does not recover, Figure 6.

\section{Discussion}

There are many models of the evolution of cooperation in group-structured populations in the literature where group selection is not a potent force, starting with the classic "haystack model", Maynard-Smith, 1964. However the Haystack model, and other models that do not feature fission and extinction events, neglect a powerful catalyst; one that is common in hunter-gatherer tribes, host/parasite populations, primitive multicellular organisms, and many other examples of group-structured populations. We have shown (at least for our hunter-gatherer model) that group selection, measured by the net effect of group-level events, can be decisive. The way group selection works in our PDE models is very different from the mechanisms studied by the classic models. In PDE models, like our hunter-gatherer model, group selection works by subtle interactions between the individual-level and group-level events. For example, even when fission and extinction rate functions are symmetric with respect to cooperators and defectors, fission and extinction events can still have a profound effect on the resulting evolutionary process. Fission events produce group-level diversity (in group size and composition), the group extinction process selects on that diversity, and this catalyzes the evolution of cooperation. In particular, since small cooperative groups grow faster than small uncooperative groups in our model, they are more likely to escape the extinction process which targets smaller groups. This can be thought of as a kind of selective extinction, which arises as an emergent property of the population dynamics. This 
mechanism also explains the counter-intuitive fact that associative splitting (segregation of cooperators and defectors in the fission event) can hinder the evolution of cooperation in the model, since cooperators often find themselves in small groups that are vulnerable to extinction.

Our results suggest that fission and extinction events might have been important factors in the evolution of cooperation in pliocene hunter-gatherer populations. However, group-level events may have been even more important for the evolution of cooperation in other examples of groupstructured populations, like host/parasite and microbial populations, where cooperator-friendly mechanisms like reciprocal altruism and gene-culture co-evolution, Richerson and Boyd, 2005, were obviously not possible.

In particular, the social microbe, Dictyostelium, is an important and well studied example of a group structured population (Strassmann, et.al., 2000; West, et.al., 2006) that bares little resemblance to hunter-gatherers. A continuous-time (PDE) model of Dictyostelium populations would feature group-level events, like dispersion and fusion (defined in Simon, Fletcher, and Doebeli, 2013; Simon, 2014), that do not appear in our hunter-gatherer model. This alters $g_{t}$ on the right side of (1), but does not alter the qualitative form of the equation. Likewise, even the highly non-human birth and death rate functions of individual amoebae within the multi-cellular stages of the Dictyostelium life cycle do not change the structure of (1). However, there is at least one important difference between populations of amoebae and hunter-gatherers: The former exist as singletons as part of their life cycles, while humans do not (at least in the model). This implies that Dictyostelium models need to include separate equations for the populations of spores and unicellular amoebae, that are then coupled to the main PDE. Given that Dictyostelium can be studied in the laboratory, it may be possible to construct accurate PDE-like models of its evolution.

\section{REFERENCES}

1. Archetti, M. and Scheuring, I., 2010. Coexistence of Cooperation and Defection in Public Goods Games, Evolution. 65-4, 1140-1148.

2. Allen. B., Nowak, M.A. and Dieckmann, U., 2013. Adaptive Dynamics with Interaction Structure. The American Naturalist. 181, E139-E163. 
3. Allen, B., and Nowak, M.A., 2015. Games among relatives revisited. Journal of Theoretical Biology. 378, 103-116.

4. Aoki, K.A., 1982. Condition for Group Selection to Prevail Over Counteracting Individual Selection. Evolution. 36, 832-842.

5. Bettinger, R., 1991. Hunter-Gatherers: Archaeological and Evolutionary Theory. Springer.

6. Bowles, S., 2006. Group Competition, Reproductive Leveling, and the Evolution of Human Altruism. (and Supporting Material) Science. 314, 1569-1572.

7. Champagnat, N., Ferriere, R., and Meleard, S., 2006. Unifying evolutionary dynamics: from individual stochastic processes to macroscopic models. Theor. Popul. Biol. 69, 297-321.

8. Damuth, J. and Heisler, I.L., 1988. Alternative Formulations of Multilevel Selection. Biology and Philosophy. 3, 407-430.

9. Darwin, C., 1859. On the Origin of Species. Cambridge: Harvard University Press.

10. Darwin, C., 1871. The Descent of Man and Selection in Relation to Sex. London: Murray, p. 166.

11. Dawkins, R., 1976. The Selfish Gene. Oxford University Press, New York.

12. Doebeil, M., Hauert, C., and Killingback, T., 2004. The Evolutionary Origin of Cooperators and Defectors. Science. 306, 859-862.

13. Eshel, I. and Cavalli-Sforza, L.L., 1982. Assortment of Encounters and the Evolution of Cooperativeness. Proc. Natl. Acad. Sci., U.S.A. 79, 1331-1335.

14. Fisher, R.A., 1930. The Genetical Theory of Natural Selection. Oxford: Clarandon Press.

15. Fletcher, J.A. and Doebeli, M., 2009. A simple and general explanation for the evolution of altruism. Proceedings of the Royal Society B. 276, 13-19.

16. Frank, S.A., 2012. Natural selection IV. The Price equation. Journal of Evolutionary Biology. 25, 1002-1019.

17. Frank, S.A., 2013. Natural selection. VII. History and interpretation of kin selection theory. Journal of Evolutionary Biology. 26, 1151-1184.

18. Gardner, A. and West, S.A., 2006. Demography, altruism, and the benefits of budding. European Society for Evolutionary Biology. 19, 1707-1716.

19. Gardner, A., West, S.A., and Wild, G., 2011. The genetical theory of kin selection. Journal of Evolutionary Biology. 24, 1020-1043.

20. Gilpin, M.E., 1975. Group Selection in Perdator-Prey Communities. Princeton University Press, Princeton, N.J.

21. Grafen, A., 1985. A geometric view of relatedness. Oxf. Surv. Evol. Biol. 2, 28-90. 
22. Grafen, A., 2006. Various remarks on Lehmann and Keller's article. Journal of Evol. Biol. 1397-1399.

23. Goodnight, C., Schwartz, J.M., and Stevens, L., 1992. Contextual Analysis of Models of Group Selection, Soft Selection, Hard Selection, and the Evolution of Altruism. American Naturalist. 140, 743-761.

24. Haldane, J.B.S., 1932. The Causes of Evolution. Harper and Brothers.

25. Hamilton, W.D., 1964. The Genetical Evolution of Social Behavior. Journal of Theoretical Biology. 7, 1-16.

26. Hamilton, W.D., 1975. Biosocial Anthropology, R. Fox, Ed. Wiley, N.Y., p. 133-155.

27. Lehmann, L., Keller, L., West, S., and Roze, D., 2007. Group selection and kin selection: Two concepts but one process. Proceedings of the National Academy of Sciences. 104, 6736-6739.

28. Lehmann, L. and Rousset, F., 2010. How life history and demography promote or inhibit the evolution of helping behaviors. Philos. Trans. R. Soc. Ser. B: Biol. Sci. 365 (1553), 2599-2617.

29. Luo, S., 2013. A Unifying Framework Reveals Key Properties of Multilevel Selection. Journal of Theoretical Biology. 341, 41-52.

30. Luo, S. and Mattingly, J.C., 2015. Scaling limits of a model for selection at two scales, arxiv [1507.00397v1].

31. Maynard-Smith, J., 1964. Group Selection and Kin Selection. Nature. 201, 1145-1146.

32. Maynard Smith, J., 1976. Group Selection. The Quarterly Review of Biology. 51, 277-283.

33. Maynard Smith, J. and Szathmary, E., 1997. The major transitions in evolution. Oxford University Press, Oxford, U.K.

34. Nowak, M.A., 2006. Evolutionary Dynamics. Harvard University Press, Cambridge.

35. Okasha, S., 2006. Evolution and the Levels of Selection. Oxford University Press.

36. Price, G.R., 1972. Extension of Covariance Selection Mathematics. Annals of Human Genetics. 35, 485-490.

37. Puhalskii, A. and Simon, B., 2012. Discrete evolutionary birth-death processes and their large-population limits. Stochastic Models. 28, 399-412.

38. Richerson, P.J. and Boyd, R., 2005. Not by Genes Alone. How Culture Transformed Human Evolution. University of Chicago Press.

39. Simon, B., 2010. A Dynamical Model of Two-Level Selection. Evolutionary Ecology Research. 12, 555-588. 
40. Simon, B., Fletcher, J.A., and Doebeli, M., 2013. Towards a general theory of group selection. Evolution. 67, 1561-1572.

41. Simon, B., Fletcher, J.A., and Doebeli, M., 2012. Hamilton's Rule in Multi-Level Selection Models. Journal of Theoretical Biology. 299, 55-63.

42. Simon, B., 2014. Continuous Time Models of Group Selection and the Dynamical Insufficiency of Kin Selection Models. Journal of Theoretical Biology. 349, 22-31.

43. Simon, B. and Nielsen, A., 2012. Numerical solutions and animations of group selection dynamics. Evolutionary Ecology Research. 14, 757-768.

44. Sober, E. and Wilson, D.S., 1998. Unto Others. Harvard University Press, Cambridge, MA.

45. Strassmann, J.E., Zhu, Y., and Queller, D.C. 2000., Altruism and social cheating in the social amoeba Dictyostelium discoideum. Nature. 408.6815, 965-967.

46. Tarnita, C.E., Taubes, C.H., and Nowak, M.A., 2013. Evolutionary construction by staying together and coming together. Journal of Theoretical Biology. 320, 10-22.

47. van Veelen, M., Garcia, J., Rand, D.G., and Nowak, M.A., 2012. Direct reciprocity in structured populations. Proceedings of the National Academy of Sciences of the USA. 109, 9929-9934.

48. van Veelen, M., Luo, S., and Simon, B., 2014. A simple model of group selection that cannot be analyzed with inclusive fitness. (Supplementary Material). Journal of Theoretical Biology. 360, 279-289.

49. Traulsen, A. and Nowak, M.A., 2006. Evolution of Cooperation by multilevel selection. Proceedings of the National Academy of Sciences of the USA. 103, 10952-10955.

50. West, S.A., Griffin, A.S., Gardner, A., and Diggle, S.P., 2006. Social evolution theory for microorganisms. Nature Reviews Microbiology. 4.8, 597-607.

51. Wilson, E.O., 1975. Sociobiology: The New Synthesis. Harvard University Press, Cambridge, MA.

52. Wilson, D.S., 1975. A Theory of Group Selection. Proceedings of the National Academy of Sciences. 72, 143-146.

53. Wilson, D.S. and Wilson, E.O., 2007. Rethinking the theoretical foundations of sociobiology. Q. Rev. Biol., 82 (4), 327-348.

54. Wright, S., 1943. Isolation by Distance. Genetics. 28, 114-138.

55. Wynne-Edwards, V.C., 1962. Animal Dispersion in Relation to Social Behavior. Oliver and Boyd, Edinburgh. 


\section{Acknowledgements}

The authors would like to thank Michael Bentley for many helpful comments, and Peter Richerson and Robert Bettinger for useful discussions on hunter-gatherer populations. The anonymous reviewers were also very helpful, and improved the resulting paper considerably. We also thank the Center for Computational Mathematics, University of Colorado Denver, for the use of the Colibri cluster, which was supported by NSF award CNS-0958354.

\section{Author Information}

Correspondence and requests for materials should be addressed to Burt. Simon@uCDenver.edu. 


\section{Methods}

Our numerical experiments involved solving the dynamical equation (1) for various settings of the model parameters. The employed numerical solution method follows a forward Euler discretization of time, and a finite volume discretization in space. The finite volume scheme insures that $G_{t}$ does not change in time when there are no group-level events, which turns out to be a crucial measure of the accuracy of the method applied to this problem. The solver is written in Matlab (ver 2012b), and does not rely on any additional packages, though the Image Toolbox is useful for visualizing the results. The program is called Catalyst.m, and can be found at https://github.com/mpilosov/cooperation along with an additional file, Visualizations.m, which allows users to recreate the figures found in the main text (generated using Matlab, with annotations being added using Adobe Photoshop). The code is commented in detail so that users can design their own experiments by changing initial conditions, group-level event rates, intragroup game dynamics, and anything else in the model. It is also possible to animate the numerical solutions with Visualizations.m. Animations are surely the best way to visualize how group-level events catalyze the evolution of cooperation in two-level population process. 


\section{Appendix}

\section{A1: Hamilton's rule for isolated groups}

We now derive Hamilton's rule for a group of cooperators and defectors, when the population within the group is governed by (8) with $\mu=0$ (no migration). Hamilton's rule is a condition for the fraction of cooperators in the population to be increasing, Hamilton, 1964; Price, 1972, which in this case means

$$
\frac{d}{d t}\left(\frac{x(t)}{x(t)+y(t)}\right)>0
$$

Simplifying (13) yields

$$
y x^{\prime}-y^{\prime} x>0
$$

From (4) and (5) we obtain

$$
y x^{\prime}=\operatorname{sxyr}(1+b-c)+\operatorname{sxy}(1-r)\left(\frac{x}{x+y}(1+b-c)+\frac{y}{x+y}(1-c)\right)-d(x+y) x y,
$$

and

$$
y^{\prime} x=\operatorname{sxyr}+\operatorname{sxy}(1-r)\left(\frac{x}{x+y}(1+b)+\frac{y}{x+y}\right)-d(x+y) x y,
$$

so $y x^{\prime}-y^{\prime} x=s x y r b-s x y c$. From (14), the fraction of cooperators in the group increases if and only if $r b>c$, regardless of the state of the group, $(x(t), y(t))$. In general, one cannot simply take model parameters called $r, b$, and $c$, and assemble them into Hamilton's rule, Grafen, 2006. However, in our hunter-gatherer model, $r$ (the level of intra-group assortment), and $b$ and $c$ (the benefit and cost terms in the prisoner's dilemma payoff matrix (2)) do combine to yield an important inequality for our hunter-gatherer model: In the absence of group-level events, cooperation evolves if and only if $r b>c$. Thus, $r^{*}=c / b$ as an important threshold: If $r<r^{*}$ then cooperation cannot evolve in the model without group-level events, and if $r>r^{*}$ then it can. Fission and extinction events change the population process considerably, so in general $r b>c$ is not the correct condition for cooperation to be favored in the population as a whole. A simple inequality, in terms of model parameters, that predicts whether or not cooperation will increase and/or thrive in the PDE model 
is unlikely to exist. See Simon, Fletcher and Doebeli, 2012, equation (22), for an example of a condition "analogous" to Hamilton's rule for group-structured populations.

\section{A2: Assigning parameter values to the hunter-gatherer model}

The hunter-gatherer model is analyzed by solving the dynamical equation (1). Anything that can be calculated from $\left\{\theta_{t}(x, y), t \geq 0\right\}$ can therefore be determined from the model. This includes important quantities like $G_{t}, C_{t}$, and $D_{t}$ from (7), or any function of them such as $C_{t} / G_{t}$, the average number of cooperators per group. Much more complicated statistics of the state of the population can also be calculated, such as measures of global assortedness like,

$$
R_{t}=P(C \mid C)-P(C \mid D)=r+(1-r)\left(E_{t}\left(\frac{X^{2}}{X+Y}\right) E_{t}(X)^{-1}-E_{t}\left(\frac{X Y}{X+Y}\right) E_{t}(Y)^{-1}\right)
$$

where $E_{t}(\cdot)$ is expected value with respect to the probability density, $\theta_{t} / G_{t}$ (also in Simon, Fletcher and Doebeli, 2012). One can also study functions of the evolutionary trajectories, like the times required for the population to reach $50 \%$ cooperators, e.g. Figures 2 and 3; or the equilibrium (limiting) population state.

However, there are some quantities of interest that cannot be directly calculated from $\left\{\theta_{t}(x, y), t \geq\right.$ $0\}$. In particular, quantities associated with specific individuals or groups, like the average individual lifetime or the average group lifetime (time until fission or extinction), are hard to determine exactly.

The parameters $\left\{b, c, d, s, e_{0}, \lambda\right\}$ are fixed in all our numerical experiments. Their values were chosen so that mean human lifetimes, mean group lifetimes, and other emergent model statistics match reasonable estimates of their true values. The model parameters (directly) specify the various rate functions (4), (5), (6), (9), (11), but not always the quantities for which we have more direct knowledge, like average group size, or number of offspring per individual. In the future it would be very useful to find rigorous methods to calculated these quantities from $\left\{\theta_{t}(x, y), t \geq 0\right\}$. However, in this study we used some simple heuristics to approximate the quantities of interest, and thereby determine the parameter settings for the baseline model. Precision was not important 
since we ran numerical experiments over wide ranges of the important parameters. What follows are "back of the envelope" calculations, which are useful for quick approximations.

Starting with the population dynamics within the groups, we can determine the approximate equilibrium group sizes from $b, c, d$, and $s$. In a group of mostly cooperators $(y \approx 0)$, the perindividual birth rate is $s \beta_{c}(x, y) \approx s(1+b-c)$ which equals 0.0325 in our benchmark model. The total birth rate in the group is therefore about $0.0325 \tau_{c}$, where $\tau_{c}$ is the equilibrium size of a group of cooperators. The per-individual death rate is $d \tau_{c}$, so in our benchmark model the total death rate in a group of cooperators is $0.0005 \tau_{c}^{2}$. Since the total birth rate and total death rate are equal in equilibrium, we obtain $\tau_{c}=65$. Likewise, a group of (mostly) defectors has a total birth rate of about $0.025 \tau_{d}$ and a total death rate of $0.0005 \tau_{d}^{2}$, so we obtain $\tau_{d}=50$. Groups with a mixture of cooperators and defectors will have an equilibrium size somewhere between 50 and 65 . This is why the diagonal line between $(0,50)$ and $(65,0)$ appears to be a boundary in Figure 2. For simplicity (precision is neither necessary nor possible in these kinds of calculations), we will use $\tau=60$ as the size of a typical mature group in our numerical experiments. Thus, the per-individual death rate in a typical group is $\tau d=0.03$, meaning that the average lifetime is about $1 / \tau d \approx 33$ time units. If we accept that hunter gatherers lived a bit more that 30 years on the average then it follows that one time unit in the model corresponds to about 1 year.

Now that we know the time unit we can estimate how often other events occur. The benchmark migration rate is $\mu=.0025$, which means that the probability an individual migrates away from the tribe in one year is about $1 / 400$. In a group of 60 this means somebody is migrating away about once every 6.6 years. (And every group can expect a migrant to join them about once every 6.6 years.)

A group of size 60 will fission at rate $60 \lambda=.015$ per time unit; in other words it will take a group about 66 years on the average to fission after it reaches full size (if the group doesn't die of extinction first). Smaller groups are less likely to fission in the model.

To estimate the average time a group lives before extinction (if it doesn't fission first) we need the equilibrium number of groups in the population. This quantity is difficult to estimate 
directly from the basic model parameters since the sizes of the groups in the population vary so much. However, we see from the numerical experiments that the equilibrium number of groups is typically around 400, which will suffice for our estimates here. If we use $G=400$ then the extinction rate of a group of 60 is about $e_{0} \cdot 400 / 60^{2}=1 / 360$, which means a mature group can expect to live about 360 years if it doesn't fission first. However, in the model a group of only 30 individuals has an extinction rate 4 times as large, and smaller groups are even more vulnerable. New groups (fissioned pieces) often start out small and therefore face the threat of extinction until they grow bigger.

The lifetime of a group is the time from when it is born until it either fissions or dies of extinction. We see that a mature group will fission after about 66 years on the average, if it doesn't go extinct first; and a mature group will die of extinction after about 360 years on the average, if it doesn't fission first. Another quantity of interest is the time it takes a fissioned piece to reach maturity. We can approximate the time using a logistic equation (since the growth is approximately logistic)

$$
P(t)=\frac{p_{e}}{1+\frac{p_{e}-p_{0}}{p_{0}} e^{-s t}},
$$

where $p_{e}$ is the equilibrium population, $p_{0}$ is the initial population, and $s=.025$ is the birth rate scaling parameter. If we use $p_{e}=60$, as we do above, then a group of size $p_{0}=25$ needs about 64 years to reach size 50. Thus, the average time from when a new group is born from a fission event until it fissions (assuming it does not die of extinction first, and does not fission until it reaches maturity) is (roughly) the time a group of 25 takes to grow to size 50, plus the time a mature group takes to fission, which is $64+66=130$ years. 


\section{FIGURES}

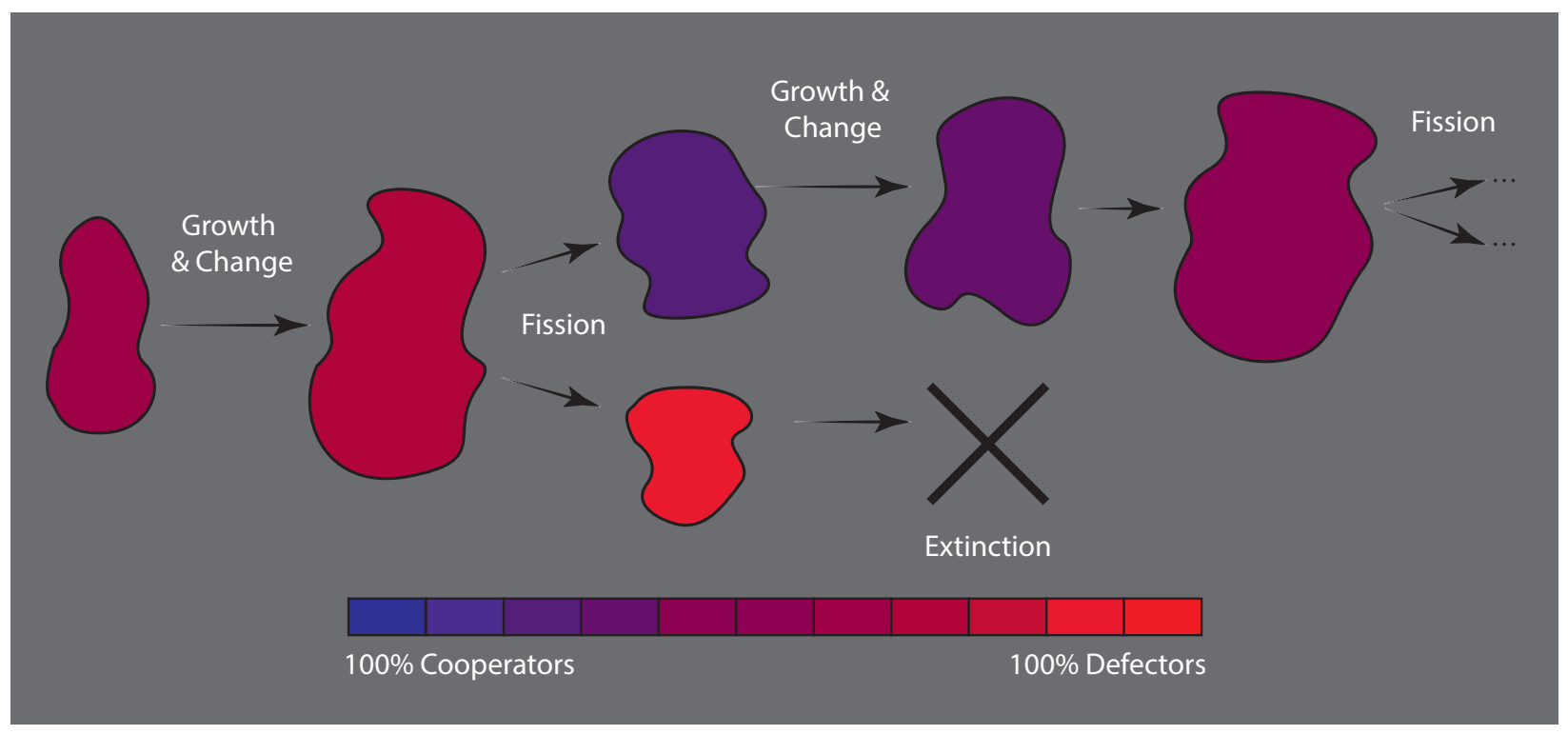

Figure 1: The Fission-Growth-Extinction Process: This diagram illustrates the case where $r b<c$ so in each group the fraction of cooperators decreases in time. However, when groups fission, the pieces typically differ in size and level of cooperation (either due to randomness, or as a result of the individuals' choices). If more-cooperative pieces have sufficient advantages over less-cooperative pieces (e.g. they grow faster or are less prone to extinction), then cooperators can thrive in the population despite their disadvantage within each group. 

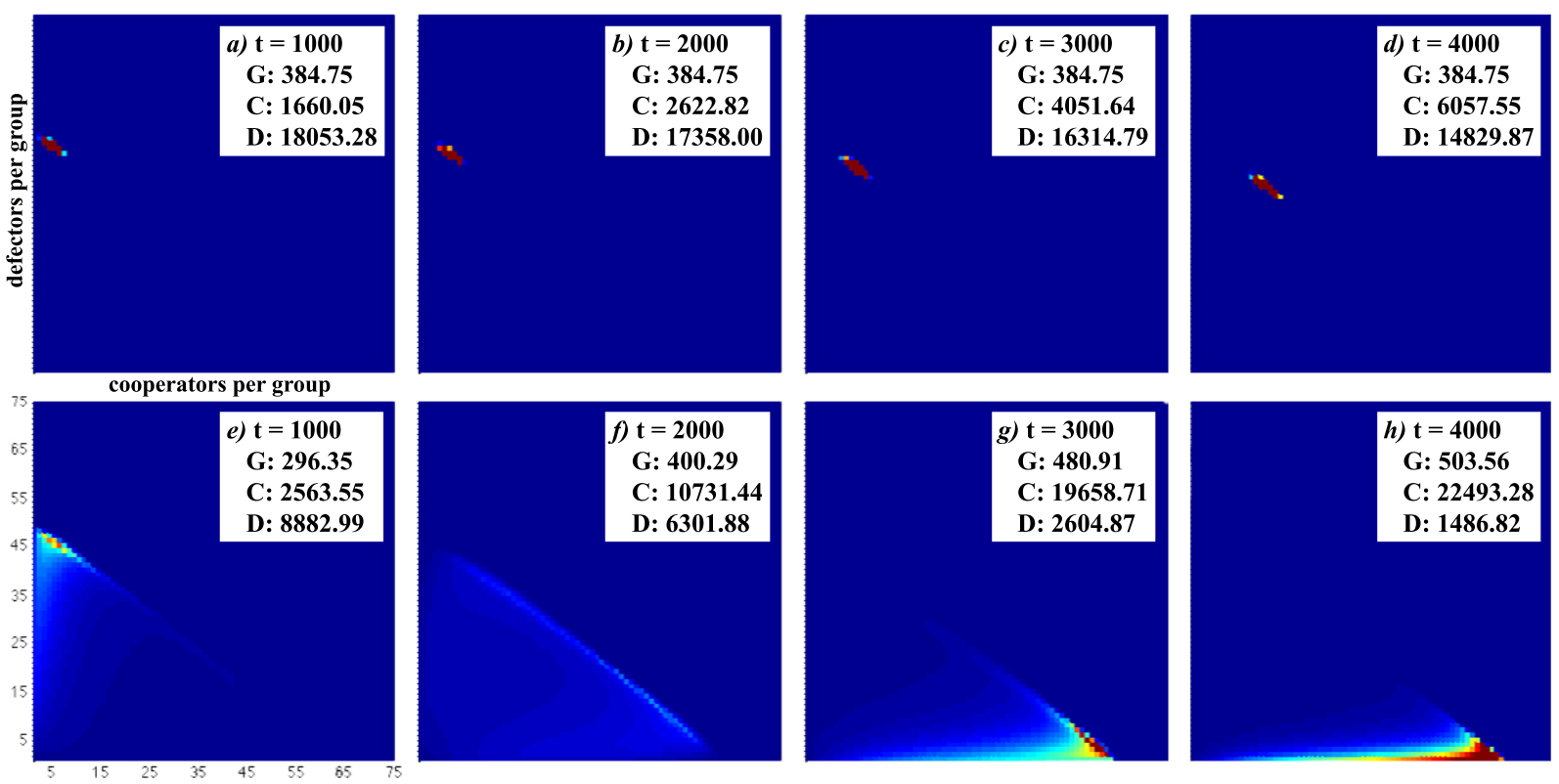

Figure 2: The Catalytic Process: The panels show $\theta_{t}(x, y), t>0$, viewed from above. The top row has $\rho=0$ (no group-level events) with $r=0.3$ and $c / b=0.25$, so cooperators are favored within the groups by Hamilton's rule. Starting with a population of about $385(2,48)$-groups, the states of the groups move in unison (slowly) down the diagonal line corresponding to equilibrium sized groups, towards the cooperative states. The number of groups stays constant since there are no group-level events. The bottom row shows the same example at the same time epochs, except that fission and extinction events occur at the benchmark rates $(\rho=1.0)$. Due to the group-level events, the population takes a "shortcut" to the cooperative states, and gets there in much less time. If $r$ was below Hamilton's threshold in this example, the population in the top row would move in the other direction, pressing against the $y$ axis, as the cooperators go extinct. However, the bottom row would look very similar (unless $r \approx 0$ ) since $\rho=1.0$ is sufficient to catalyze the individual-level process (Figure 3). There are links to full animations of the evolutionary trajectories for these and other examples in the Methods section. 

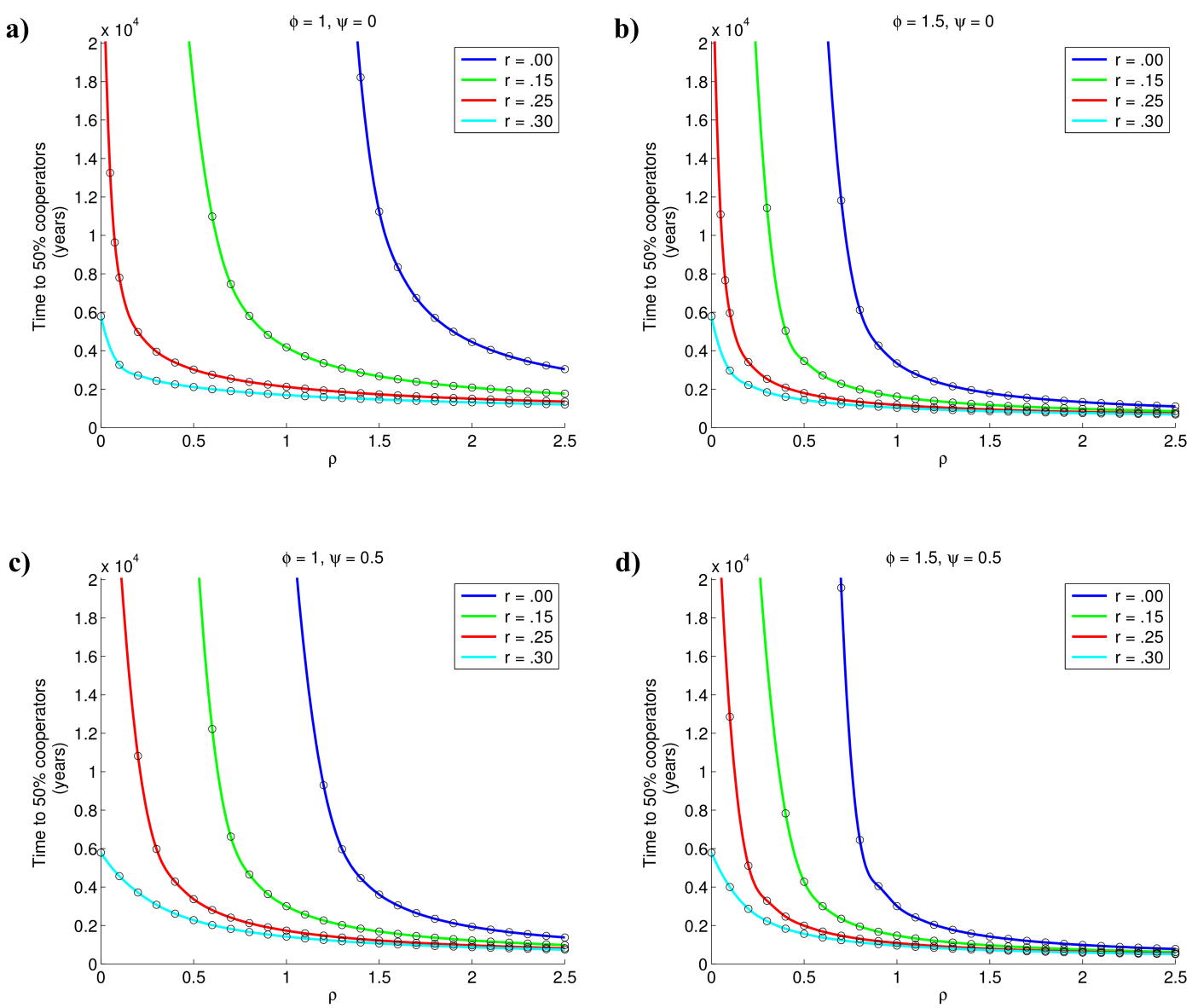

Figure 3: The effect of group-level event rates: This figure shows how long it takes an initially uncooperative population (5\% cooperators) to reach 50\% cooperators, as functions of $\rho$ and $r$ for various amounts of selective extinction (controlled by $\phi$ ) and associative splitting (controlled by $\psi$ ). $\psi=0$ and $\phi=1$ are the neutral settings. When $r=.3$ (cyan lines), cooperators reach 50\% (and beyond) even if $\rho=0$ since $r b>c$. However, introducing group-level events $(\rho>0)$ speeds the process up significantly. When $r<c / b=.25$, there is a threshold value of $\rho$, so that below that value the fraction of cooperators never reaches 50\%. Above the threshold, further increasing $\rho$ greatly speeds up the process. In our model, cooperation evolves easily even without selective extinction and associative splitting (top left panel) since the natural variability in the fission event is enough to catalyze the process. The small circles on the curves correspond to numerical solutions of (1). 

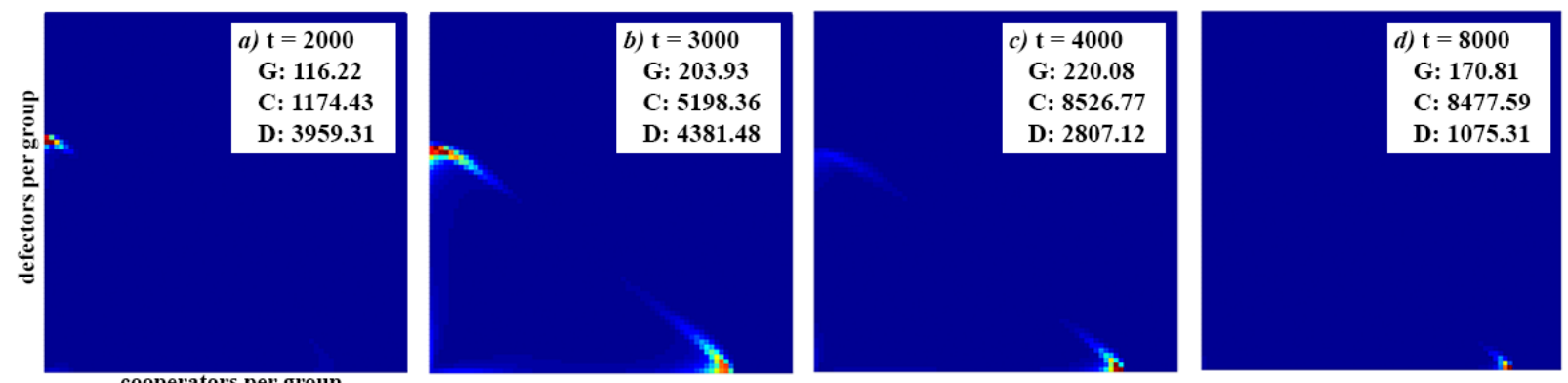

Figure 4: Associative splitting does not always benefit cooperators: This figure shows what can happen in the hunter-gatherer model when there is an extreme form of associative splitting. In this example, fission events always result in the perfect segregation of cooperators and defectors into different groups, i.e. $h((x, y),(u, v))=$ $\delta_{((x, y)=(u, 0))}+\delta_{((x, y)=(0, v))}$. Before long, it is only migration that prevents the groups from being monomorphic. Since fissioning is not an useful method of group reproduction in this case, the whole population eventually dies out. 
a)

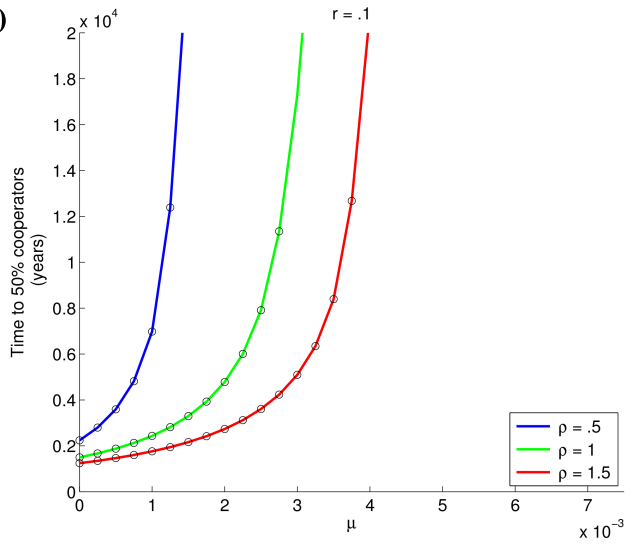

c)

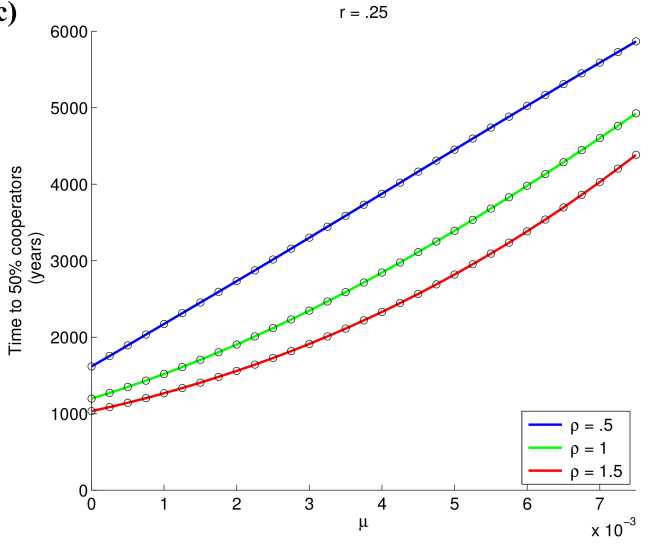

b)

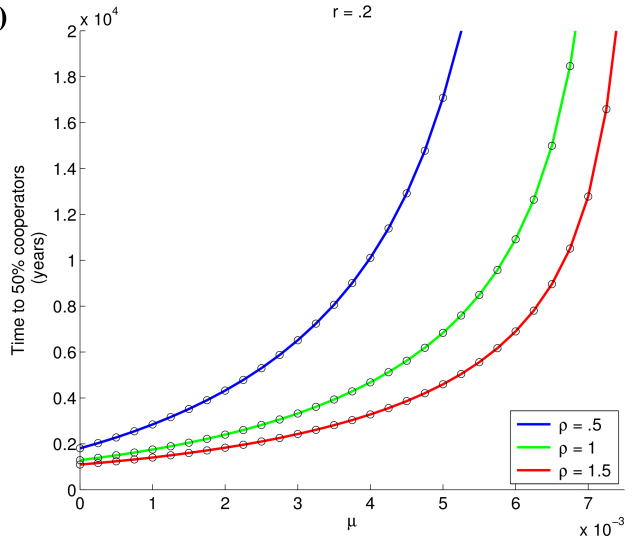

d)

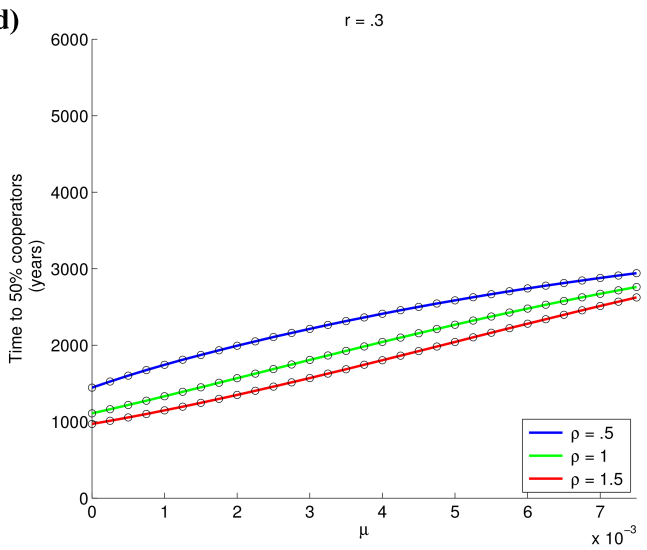

Figure 5: The effect of migration on the evolution of cooperation: This figure shows how long it takes for an initially uncooperative population ( $5 \%$ cooperators) to reach $50 \%$ cooperators as a function of the migration rate for various combinations of $r$ and $\rho$. Clearly migration always makes the evolution of cooperation more difficult, but it is not necessarily fatal. Even when $r<c / b=0.25$ (top row) cooperators thrive if $\mu$ is below a threshold. The threshold (of course) depends on $\rho$. The benchmark migration rate of $\mu=.0025$ corresponds to an individual migrating to or from each (equilibrium-sized) group every 3.3 years on the average (see Appendix A1). When $r \geq 0.25$ (bottom row), even high rates of migration (beyond the range seen here), cannot prevent the evolution of cooperation. The small circles on the curves correspond to numerical solutions of (1). 

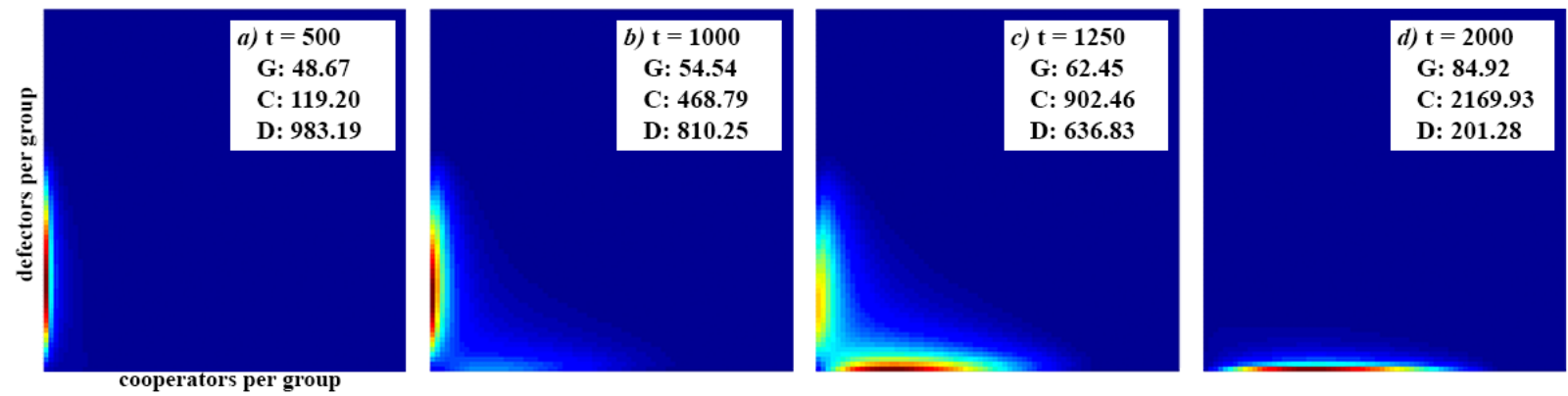

Figure 6: The effect of too many group-level events: Group-level events, in moderation, catalyze the evolution of cooperation, but too many group-level events can be counterproductive. Intuituively, too many fission events cause the groups to break into smaller and smaller pieces, which are more vulnerable to extinction events. In this example, $\rho=$ 5.0, which is five times the benchmark level. There is a mass die-off of groups when the process starts $\left(G_{0}=384.75\right.$, $G_{500}<50$ ), which stabilizes and starts to recover when the first small cooperative groups emerge at around $t=1000$, but the population never reaches the numbers we see in the benchmark model. Cooperators dominate defectors in equilibrium, but the equilibrium is a mixture of relatively small groups. (Compare to figure 2.) 